\title{
Matter Density Distributions, Root-mean Square Radii and Elastic Electron Scattering Form Factors of Some Exotic Nuclei $\left({ }^{17} \mathrm{~B},{ }^{11} \mathrm{Li},{ }^{8} \mathrm{He}\right)$
}

\author{
Shaimaa A. Rahi ${ }^{\text {a }}$, Ghaith N. Flaiyh* \\ Department of Physics, College of Science, University of Baghdad, Baghdad, Iraq \\ ${ }^{a}$ E-mail: shimaa.rahi1204@sc.uobaghdad.edu.iq \\ *Corresponding author: kaithnaima@yahoo.com
}

\begin{abstract}
The two-neutron halo-nuclei $\left({ }^{17} \mathrm{~B},{ }^{11} \mathrm{Li},{ }^{8} \mathrm{He}\right)$ was investigated using a two-body nucleon density distribution (2BNDD) with two frequency shell model (TFSM). The structure of valence two-neutron of ${ }^{17} \mathrm{~B}$ nucleus in a pure $\left(1 \mathrm{~d}_{5 / 2}\right)$ state and in a pure $\left(1 \mathrm{p}_{1 / 2}\right)$ state for ${ }^{11} \mathrm{~L}$ and ${ }^{8} \mathrm{He}$ nuclei. For our tested nucleus, an efficient (2BNDD's) operator for point nucleon system folded with two-body correlation operator's functions was used to investigate nuclear matter density distributions, root-mean square (rms) radii, and elastic electron scattering form factors. In the nucleon-nucleon forces the correlation took account of the effect for the strong tensor force (TC's). The wave functions of single particle harmonic oscillator are used with two different oscillator size parameters $\beta \mathrm{c}$ and $\beta \mathrm{v}$, where the former is for the core (inner) orbits and the latter is for the valence (halo) orbits. The measured matter density distributions of these nuclei clearly showed long tail results. To investigate elastic electron scattering form factors the plane wave born approximation (PWBA) with two body nucleon density distribution (2BNDD's) was use.
\end{abstract}

Article Info.

\section{Introduction}

Over the last two decades, the world of atomic nuclei has changed dramatically and continues to do so. This is partially because the nuclear structure physics frontier has moved from stable to exotic nuclei. Exotic nuclei are atomic nuclei with shorter life times and in most situations, an unbalanced ratio between the neutron number $(\mathrm{N})$ and the proton number $(\mathrm{Z})$, whereas stable nuclei are atomic nuclei with sufficiently long or infinite life time's [1]. Tanihata et.al with his experiment's to measure the radius of the ${ }^{11} \mathrm{Li}$ nucleus using RI (radioactive ion or rare isotope) beams of this nucleus in 1985 was the first step in such developments. According to this experiment, ${ }^{11} \mathrm{Li}$ has a very high interaction cross section [2]. Many new progresses have been made in recent years. The discovery of halo phenomena in exotic nuclei is one of the typical examples. In general, the halo nucleus has large neutron or proton excess where a few outside nucleons are very weakly bound. Such halo systems are well described by the few-body models, which assume that halo nuclei consist of a core and a few outside nucleons [3]. While the inclusion of the strong tensor force is one of the most basic nuclear forces, its firstorder impact on the shell structure has only recently been explained in exotic nuclei studies Depending on the occupation of specific orbits, the tensor force will alter the spin-orbit splitting [1]. T. Frederico et al. [4] used zero-range interactions to study the structure of three-body halo nuclei produced by two neutrons and a core. Only the swave scattering lengths and two-neutron separation energy will fully parameterize the halo wave function. Low-energy properties of the one- and two-neutron halo of light 
exotic nuclei, which are dominated by $s$-wave short-range two-body interactions were studied by T. Frederico, et al. [5]. In the study of Raghad Imad, showed that the twobody tensor correlations exhibit a mass dependence due to the strength parameter $\alpha$ (A), while the two-body short range correlations did not exhibit this dependency [6]. The inelastic longitudinal form factors $\mathrm{C} 2$ determined using this transition charge density with excitation of the levels for ${ }^{54,52,50} \mathrm{Cr}$ nuclei was shown by G.N. Flaiyh [7]. The central polarization transition density is calculated using the Tassie model's shape and the derived form of the ground state two-body charge density distributions (2BCDD's) in his research. Ahmed N. Abdullah [8] studied the nuclear matter distributions of neutron rich ${ }^{6} \mathrm{He},{ }^{11} \mathrm{Li},{ }^{14} \mathrm{Be}$ and ${ }^{17} \mathrm{~B}$ halo nuclei by the Bear Hodgson potential. These halo nuclei are treated as a three-body system composed of core and outer two-neutron (Core $+n+n$ ). The radial wave functions of the Bear-Hodgson potential are used to describe the core and halo density distributions. The interaction of core-neutron takes the Bear-Hodgson potential form. The outer two neutrons of ${ }^{6} \mathrm{He}$ and ${ }^{11} \mathrm{Li}$ interact by the realistic interaction REWIL whereas those of ${ }^{14} \mathrm{Be}$ and ${ }^{17} \mathrm{~B}$ interact by the realistic interaction of HASP. The obtained results showed that this model succeeds in reproducing the neutron halo in these nuclei.

The aim of the work is to derive an expression for the ground state two - body nucleon density distributions (2BNDD's) of some exotic nuclei $\left({ }^{17} \mathrm{~B},{ }^{11} \mathrm{Li},{ }^{8} \mathrm{He}\right)$, based on the use of the two - body wave functions of the harmonic oscillator in order to employ it for the study of the effects of the (TC's), and oscillator size parameter on the root mean square radii, 2BNDD's and elastic form factors.

\section{Theory}

The following transformation could be used to convert the one-body density operator to a two-body density type [9].

$$
\begin{gathered}
\hat{\boldsymbol{\rho}}^{(1)}(\overrightarrow{\mathrm{r}})=\sum_{\mathrm{i}=1}^{\mathrm{A}} \boldsymbol{\delta}\left(\overrightarrow{\mathrm{r}}-\overrightarrow{\mathrm{r}}_{\mathrm{i}}\right) \\
\hat{\boldsymbol{\rho}}^{(1)}(\overrightarrow{\mathrm{r}}) \Rightarrow \hat{\boldsymbol{\rho}}^{(2)}(\overrightarrow{\mathrm{r}})
\end{gathered}
$$

i.e

$$
\sum_{\mathrm{i}=1}^{\mathrm{A}} \boldsymbol{\delta}\left(\overrightarrow{\mathrm{r}}-\overrightarrow{\mathrm{r}}_{\mathrm{i}}\right) \equiv \frac{1}{2(\boldsymbol{A}-1)} \sum_{\mathrm{i} \neq \mathrm{j}}\left\{\delta\left(\overrightarrow{\mathrm{r}}-\overrightarrow{\mathrm{r}}_{\mathrm{i}}\right)+\boldsymbol{\delta}\left(\overrightarrow{\mathrm{r}}-\overrightarrow{\mathrm{r}}_{\mathrm{j}}\right)\right\}
$$

In reality, a useful transformation of the coordinates of the two particles, $\vec{r}_{i}$ and $\overrightarrow{\mathrm{r}}_{\mathrm{j}}$, to be in terms of that center - of -mass $\vec{R}_{\mathrm{ij}}$ and relative $\overrightarrow{\mathrm{r}}_{\mathrm{ij}}$ coordinates, can be rendered [10].

$$
\begin{aligned}
& \overrightarrow{\mathbf{r}}_{\mathrm{ij}}=\frac{1}{\sqrt{2}}\left(\overrightarrow{\mathbf{r}}_{\mathrm{i}}-\overrightarrow{\mathbf{r}}_{\mathrm{j}}\right) \\
& \vec{R}_{\mathrm{ij}}=\frac{1}{\sqrt{2}}\left(\overrightarrow{\mathbf{r}}_{\mathrm{i}}+\overrightarrow{\mathbf{r}}_{\mathrm{j}}\right)
\end{aligned}
$$

Subtracting and adding (3a) and (3b) we obtain

$$
\begin{aligned}
& \overrightarrow{\mathbf{r}}_{\mathrm{i}}=\frac{1}{\sqrt{2}}\left(\vec{R}_{\mathrm{ij}}+\overrightarrow{\mathbf{r}}_{\mathrm{ij}}\right) \\
& \overrightarrow{\mathbf{r}}_{\mathrm{j}}=\frac{1}{\sqrt{2}}\left(\vec{R}_{\mathrm{ij}}-\overrightarrow{\mathbf{r}}_{\mathrm{ij}}\right)
\end{aligned}
$$


Introducing Eq. (3c) and (3d) into Eq. (2) yields:

$$
\hat{\rho}^{(2)}(\overrightarrow{\mathbf{r}})=\frac{\sqrt{2}}{(A-1)} \sum_{\mathrm{i} \neq \mathrm{j}}\left\{\delta\left[\sqrt{2} \overrightarrow{\mathrm{r}}-\vec{R}_{\mathrm{ij}}-\overrightarrow{\mathrm{r}}_{\mathrm{ij}}\right]+\delta\left[\sqrt{2} \overrightarrow{\mathrm{r}}-\vec{R}_{\mathrm{ij}}+\overrightarrow{\mathrm{r}}_{\mathrm{ij}}\right]\right\}
$$

Finally, by folding the operator of Eq. (4) with the two-body correlation functions $\widetilde{f}_{i j}$, the effective two-body nucleon density operator (to be used with uncorrelated wave functions) is produced:

$$
\hat{\rho}_{e f f}^{(2)}(\overrightarrow{\mathbf{r}})=\frac{\sqrt{2}}{2(A-1)} \sum_{\mathrm{i} \neq \mathrm{j}} \tilde{f}_{i j}\left\{\delta\left[\sqrt{2} \overrightarrow{\mathbf{r}}-\vec{R}_{\mathrm{ij}}-\overrightarrow{\mathbf{r}}_{\mathrm{ij}}\right]+\delta\left[\sqrt{2} \overrightarrow{\mathbf{r}}-\vec{R}_{\mathrm{ij}}+\overrightarrow{\mathbf{r}}_{\mathrm{ij}}\right]\right\} \tilde{f}_{i j}
$$

In this paper, we will use a simple model type of the two-body tenser correlation operators from [11],

i.e. $\quad \tilde{f}_{i j}=\left\{1+\alpha(\mathrm{A}) S_{i j}\right\} \Delta_{2}$

The two-body tensor correlations (TC's) presented in Eq. (6), which are caused by the strong tensor factor in the nucleon-nucleon force and have a longer range. Here $\Delta_{2}$ is a projection operator onto the ${ }^{3} S_{1}$ and ${ }^{3} D_{1}$ states only. However, Eq. (6) can be rewritten as:

$$
\tilde{f}_{i j}=\sum_{\gamma}\left\{1+\alpha_{\gamma}(\mathrm{A}) S_{i j}\right\} \Delta_{\gamma}
$$

where the sum $\gamma$, in Eq. (7), is over all reaction channels, $S_{i j}$ is the usual tensor operator, formed by the scalar product of a second-rank operator in intrinsic spin-space and coordinate space and is defined by

$$
S_{i j}=\frac{3}{r_{i j}^{2}}\left(\vec{\sigma}_{i} \cdot \vec{r}_{i j}\right)\left(\vec{\sigma}_{j} \cdot \vec{r}_{i j}\right)-\vec{\sigma}_{i} \cdot \vec{\sigma}_{j}
$$

while $\alpha_{\gamma}(\mathrm{A})$ is the strength of tensor correlations and it is non zero only in the ${ }^{3} S_{1}-{ }^{3} D_{1}$ channels.

As the halo nuclei is oversized and easily broken system consisting of a compact core plus a number of outer nucleons loosely bound and specially extended far from the core, it is suitable to separate the ground state density distribution of Eq. (5) into two parts, one is connected with the core nucleons and the other one with the halo nucleons, so the matter density distribution for the whole halo nucleus becomes [12]:

$$
\rho_{m}(r)={ }^{\text {core }} \rho_{p+n}(r)+{ }^{\text {valance }} \rho_{p(n)}(r)
$$

The normalization condition of the above ground state densities is given by:

$$
g=4 \pi \int_{0}^{\infty} \rho_{g}(r) r^{2} d r
$$

One of the following densities is represented by $\rho_{\mathrm{g}}(\mathrm{r})$ matter, charge, center, and halo densities the rms radii of the above densities are calculated as follows:

$$
\langle r\rangle_{g}^{1 / 2}=\frac{4 \pi}{g} \int_{0}^{\infty} \rho_{g}(r) r^{4} d r
$$


The cross section for elastic electron scattering form factor from spin zero nuclei $(J=0)$, can be determined by the ground - state charge density distributions (CDD). In the Plane Wave Born Approximation (PWBA), the incident and scattered electron waves are considered as plane waves and the CDD is real and spherical symmetric, therefore the form factor is simply the Fourier transform of the CDD. Thus $[13,14]$.

$$
\boldsymbol{F}(\boldsymbol{q})=\frac{4 \pi}{q Z} \int_{0}^{\infty} \rho_{o}(\mathbf{r}) \sin (q \mathbf{r}) \mathbf{r} d \mathbf{r} \boldsymbol{F}_{f s}(q) F_{c m}(q)
$$

where $F_{f s}(q)$ the finite nucleon size and $F_{c m}(q)$ the center of mass corrections. $F_{f s}(q)$ is considered as free nucleon form factor and assumed to be the same for protons and neutrons. This correction takes the form [14].

$$
F_{f s}(q)=e^{-0.43 q^{2} / 4}
$$

The correction $F_{c m}(q)$ removes the spurious state arising from the motion of the center of mass when shell model wave function is used and given by [13]:

$$
\boldsymbol{F}_{c m}(q)=e^{q^{2} b^{2} / 4 A}
$$

where $\mathrm{A}$ is the nuclear mass number.

\section{Results and discussion}

The nuclear ground state properties of two-neutron $\left({ }^{17} \mathrm{~B},{ }^{11} \mathrm{Li},{ }^{8} \mathrm{He}\right)$ exotic nuclei was calculated using (2BNDD'S) including the effect of two-body tenser correlations (TC's) using two frequency shell model (TFSM). Different model spaces for the core and the extra halo nucleon were used in the calculations. The wave functions of a single particle harmonic oscillator were used with two different size parameters of $\beta_{\mathrm{c}}$ (size parameters of core) and $\beta_{\mathrm{v}}$ (size parameters of valence). Table 1 shows the parameters of $\beta_{\mathrm{c}}$ and $\beta_{\mathrm{v}}$ used in TFSM of the present study together with the calculated and experimental rms radii of exotic nuclei $\left({ }^{17} \mathrm{~B},{ }^{11} \mathrm{Li},{ }^{8} \mathrm{He}\right)$. It is clear from this table there was a good agreement between the calculated and experimental result of rms radii for these exotic nuclei.

\begin{tabular}{|c|c|c|c|c|c|c|c|}
\hline \multirow{2}{*}{$\begin{array}{l}\text { Halo } \\
\text { nuclei }\end{array}$} & \multirow{2}{*}{$\begin{array}{l}\text { Core } \\
\text { nuclei }\end{array}$} & \multirow{2}{*}{$\begin{array}{c}\boldsymbol{\beta}_{\boldsymbol{c}} \\
(\mathrm{fm})\end{array}$} & \multirow{2}{*}{$\begin{array}{c}\boldsymbol{\beta}_{v} \\
(\mathrm{fm})\end{array}$} & \multicolumn{2}{|c|}{$\begin{array}{c}\text { rms matter radii for core } \\
\text { nuclei }\left\langle r^{2}\right\rangle_{\text {core }}^{1 / 2} \text { (fm) }\end{array}$} & \multicolumn{2}{|c|}{$\begin{array}{c}\text { rms matter radii for halo } \\
\text { nuclei }\left\langle r^{2}\right\rangle_{\text {halo }}^{1 / 2}(\mathrm{fm})\end{array}$} \\
\hline & & & & $\begin{array}{l}\text { Calculated } \\
\text { results } \\
\text { (P. W.) }\end{array}$ & $\begin{array}{l}\text { Experimental } \\
\text { results }\end{array}$ & $\begin{array}{l}\text { Calculated } \\
\text { results } \\
\text { (P. W.) }\end{array}$ & $\begin{array}{l}\text { Experimental } \\
\text { results }\end{array}$ \\
\hline${ }^{17} \mathrm{~B}$ & ${ }^{15} \mathrm{~B}$ & 1.70 & 2.20 & 2.80 & $2.4 \pm 0.25[15]$ & 3.10 & $3.0 \pm 0.6[16]$ \\
\hline${ }^{11} \mathrm{Li}$ & ${ }^{9} \mathrm{Li}$ & 1.70 & 2.18 & 2.34 & $2.32 \pm 0.02[17]$ & 3.13 & $3.12 \pm 0.16[17]$ \\
\hline${ }^{8} \mathrm{He}$ & ${ }^{6} \mathrm{He}$ & 1.80 & 1.75 & 2.27 & $2.29 \pm 0.05[18]$ & 2.55 & $2.53 \pm 0.08[18]$ \\
\hline
\end{tabular}

Table 1: The Parameters for $\beta_{c}$ and $\beta_{v}$ utilized in the TFSM of the present study together with the calculated and experimental rms radii of exotic nuclei $\left({ }^{17} \mathrm{~B},{ }^{11} \mathrm{Li},{ }^{8} \mathrm{He}\right)$.

\section{1. ${ }^{17}$ B nucleus}

${ }^{17} \mathrm{~B}$ is two-neutron exotic nuclei. The nucleus ${ }^{17} \mathrm{~B}\left(\boldsymbol{J}^{\pi}, \boldsymbol{T}=3 / 2^{-}, 7 / 2\right)$ is formed by coupling the core ${ }^{15} \mathrm{~B}\left(J^{\pi}, T=3 / 2^{-}, 5 / 2\right)$ with the valence two-neutrons $\left(J^{\pi}, T=0^{+}, 1\right)$. The value of oscillator size parameter for core ${ }^{15} \mathrm{~B}$ is equal to $\beta \mathrm{c}=1.7 \mathrm{fm}$, which gives $\mathrm{rms}$ radii equal to $2.46 \mathrm{fm}$, while the valence (two-neutron) 
assumed to be in a pure $\left(1 \mathrm{~d}_{2 / 5}\right)$ and oscillator size parameter equal to $\beta \mathrm{v}=2.2 \mathrm{fm}$ used to give rms radii equal to $(2.80 \mathrm{fm})$. The experimental and calculated nucleon rms radii for this nucleus are displayed in (Table 1). It was noted that there is good agreement between the theoretically calculated values of rms radii with the experimental data. Two body nucleon density distribution (2BNDD'S) in $\mathrm{fm}^{-3}$ of ground state were plotted versus $\mathrm{r}$ (in fm) as shown in Fig.1(a), the black line represents the normal contribution of core ${ }^{15} \mathrm{~B}$, the valence (two-neutron in state $1 \mathrm{~d}_{2 / 5}$ ) is represented by the blue line, which takes the shape of a long tail in this distribution. The matter density distribution (core + valence) is represented by a red line, which has a long tail as well and agrees well with ${ }^{17}$ B's experimental data, which were taken from Vagen et al. [19] and represented by the shaded space. Fig. 1(b) shows a comparison between the matter density distribution of ${ }^{17} \mathrm{~B}$ (represented by the red line) and the matter density distribution of stable nuclei ${ }^{10} \mathrm{~B}$ (represented by the blue line). It is clear from this figure that the red and blue curves are diverse. As the valence two neutrons in ${ }^{17} \mathrm{~B}$ is weakly bounded, the red curve has a longer tail than that of the blue curve. Fig. 1 ( $a$ and b) provide the conclusion that the halo phenomenon in ${ }^{17} \mathrm{~B}$ is connected to the valence neutrons but not to the core nucleons.

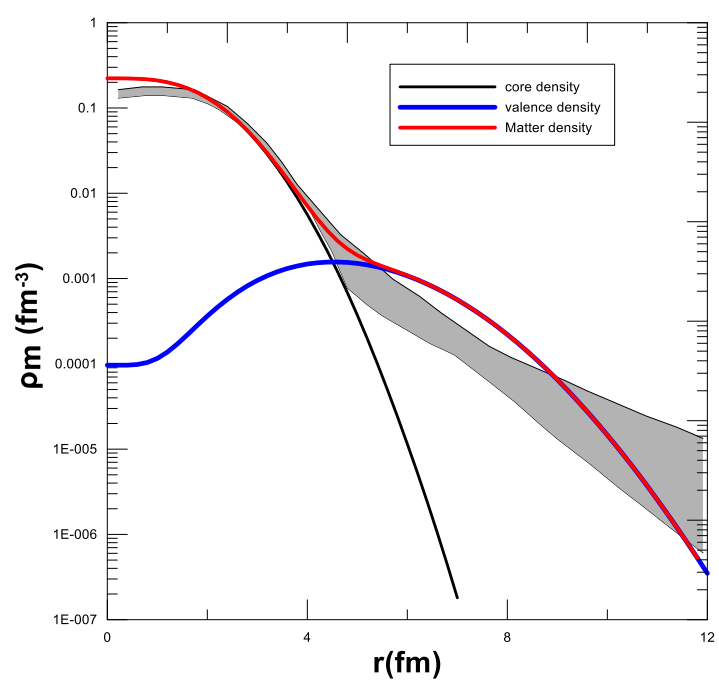

(a)

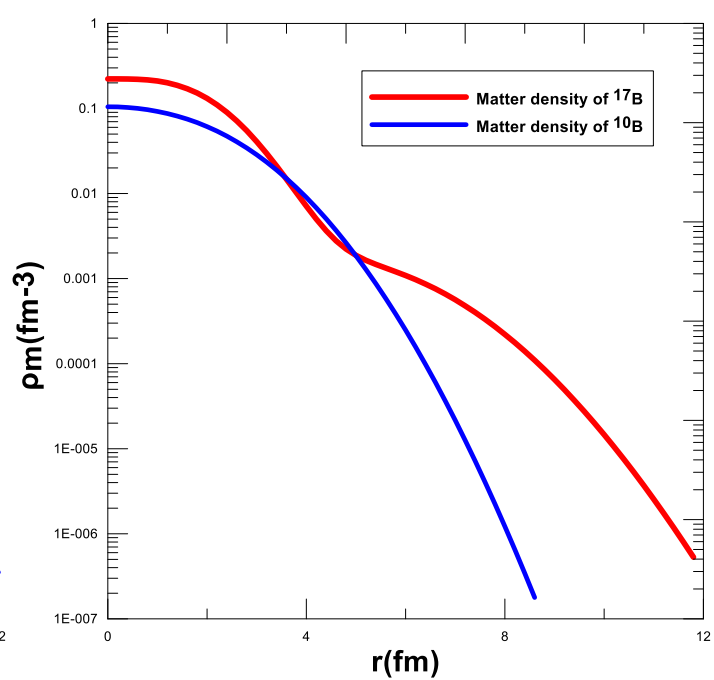

(b)

Figure 1: (a) The comparison between Matter density distributions of ${ }^{17} B$ with that of experimental data, (b) The comparison between matter density distribution of ${ }^{17} B$ and ${ }^{10} B$.

The elastic form factor are shown in the Fig.2 the red curve represents form factor with Oscillator size parameter $(\beta=1.95 \mathrm{fm})$ (assumed to be the average of $\beta_{\mathrm{c}}$ and $\beta_{\mathrm{v}}$ ), while the line with the black filled circles represents the experimental data for stable nuclei ${ }^{10} \mathrm{~B}$ which were taken from Cichocki et al. [20]. When comparing the theoretical result with the experimental data of elastic electron scattering form factor, a different behavior between exotic nuclei $\left({ }^{17} \mathrm{~B}\right)$ and stable nuclei $\left({ }^{10} \mathrm{~B}\right)$ was noted, where the first diffraction minimum for ${ }^{17} \mathrm{~B}$ located at $q \approx 1.7 \mathrm{fm}^{-1}$.

\section{2. ${ }^{11} \mathrm{Li}$ nucleus}

The second nucleus studied in this work was ${ }^{11} \mathrm{Li}$. The nucleus ${ }^{11} \mathrm{Li}$ $\left(J^{\pi}, T=3 / 2^{-}, 5 / 2\right)$ is formed by coupling the core ${ }^{9} \mathrm{Li}\left(J^{\pi}, T=3 / 2^{-}, 3 / 2\right)$ with valence two-neutron $\left(J^{\pi}, T=0^{+}, 1\right)$ the oscillator size parameter of core is equal to $\beta c=1.70 \mathrm{fm}$, Which gives rms radii equal to $(2.34 \mathrm{fm})$, while the valence (two-neutron) is assumed to be in a pure $\left(1 \mathrm{p}_{1 / 2}\right)$ with occupation number 0.5 and oscillator size parameter equal to $2.18 \mathrm{fm}$ which gives rms radii equal to $3.13 \mathrm{fm}$. The experimental 
and calculated nucleon rms radii for this nucleus are displayed in Table 1. A good agreement between the theoretically calculated and the experimental results was noted.

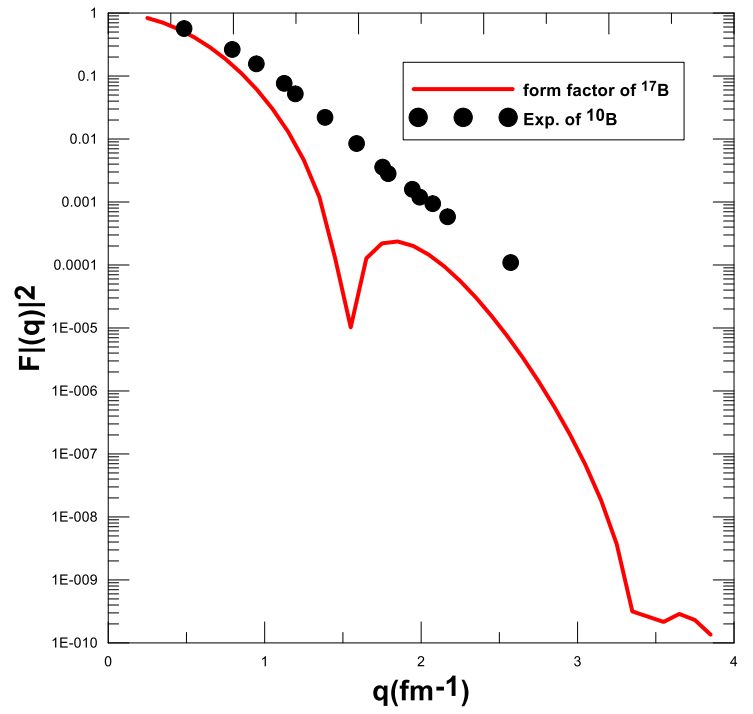

Figure 2: Comparison between the calculated elastic form factors of ${ }^{17} B$ and the experimental data of ${ }^{10} \mathrm{~B}$.

Two body nucleon density distribution (2BNDD'S) in $\mathrm{fm}^{-3}$ of ground state are plotted versus $\mathrm{r}$ (in fm) as shown in Fig.3(a), the black line represents the normal contribution of core ${ }^{9} \mathrm{Li}$, The valence (two-neutron exotic nuclei in the state of $1 \mathrm{p}_{1 / 2}$ ) is represented by the blue line, which takes the shape of a long tail in this distribution, the matter density distribution (core + valence) is defined by a red line, which has a long tail as well and agrees well with ${ }^{11} \mathrm{Li}$ 's experimental data, which were taken from Dobrovolsky [21] and is represented by the shaded space. Fig. 3(b) shows a comparison between the matter density distribution of ${ }^{11} \mathrm{Li}$ which is represented by the red line, and the matter density distribution of stable nuclei ${ }^{7} \mathrm{Li}$ represented by the blue line. It is clear from this figure that the red and blue curves are diverse. As the valence two neutrons in ${ }^{11} \mathrm{Li}$ are weakly bound, the red curve has a longer tail than that of the blue curve. Fig. $3\left(\mathrm{a}\right.$ and $\mathrm{b}$ ) provide the conclusion that the halo phenomenon in ${ }^{11} \mathrm{Li}$ is connected to the valence neutrons but not to the core nucleons.

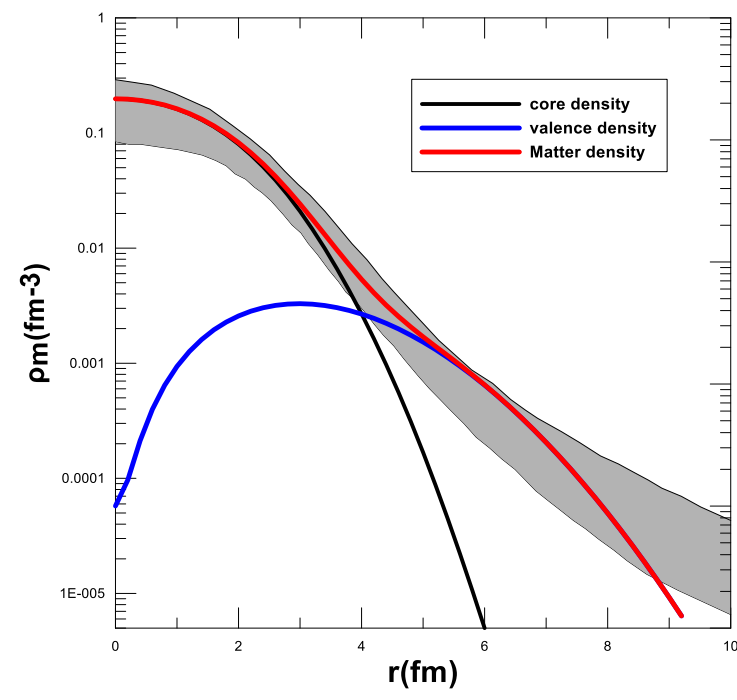

(a)

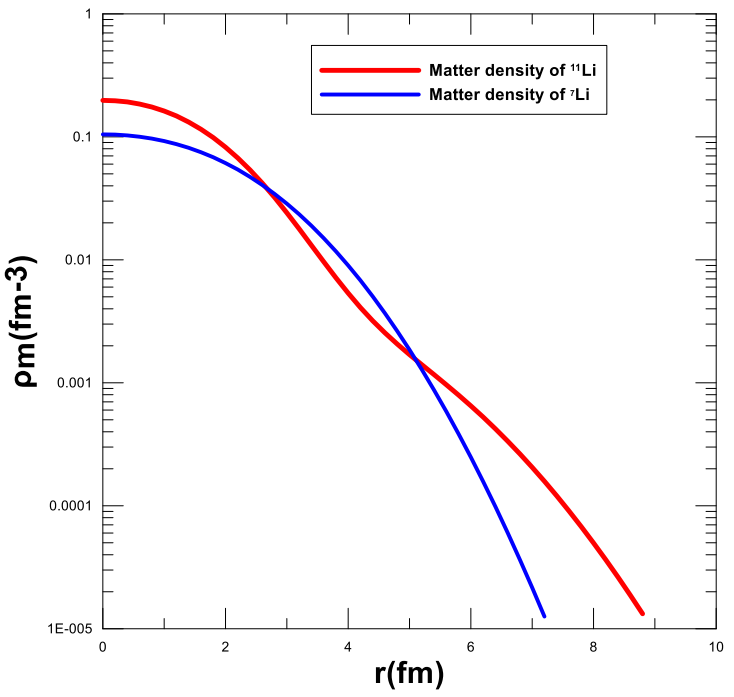

(b)

Figure 3: (a) The comparison between matter density distributions of ${ }^{11}$ Li with that of experimental data, (b) The comparison between matter density distribution of ${ }^{11} \mathrm{Li}$ and ${ }^{7} \mathrm{Li}$. 
The elastic electron scattering form factor 2BCDD's are shown in Fig.4, the red curve represents form factor with oscillator size parameter $(\beta=1.94 \mathrm{fm})$ (assumed to be the average of $\beta_{\mathrm{c}}$ and $\beta_{\mathrm{v}}$ ), while the curve with black filled circles represents the experimental data for stable nuclei ${ }^{7} \mathrm{Li}$, which were taken from Suelzle et al. [22]. When comparing the theoretical results with experimental data, it was noted that they have the same behavior of first diffraction minimum, where the first diffraction minimum for ${ }^{11} \mathrm{Li}$ is located at $q \approx 1.6 \mathrm{fm}^{-1}$ and for ${ }^{7} \mathrm{Li}$ at $q \approx 2.5 \mathrm{fm}^{-1}$.

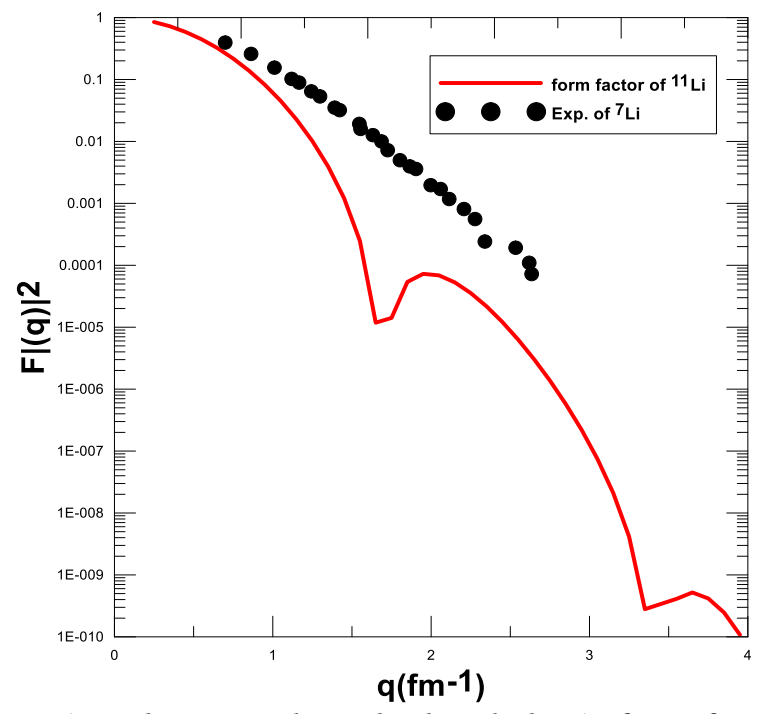

Figure 4: Comparison between the calculated elastic form factors of ${ }^{11} \mathrm{Li}$ and the experimental data of stable ${ }^{7} \mathrm{Li}$ nuclei.

\section{3. ${ }^{8}$ He nucleus}

The third nucleus are studied in this work ${ }^{8} \mathrm{He}$. The nucleus ${ }^{8} \mathrm{He}\left(J^{\pi}, T=0^{+}, 2\right)$ is formed by coupling the core ${ }^{6} \mathrm{He}\left(J^{\pi}, T=0^{+}, 1\right)$ with valence (two-neutron) $\left(J^{\pi}, T=0^{+}, 1\right)$. The oscillator size parameter of core is equal to $\beta \mathrm{c}=1.80 \mathrm{fm}$, which gives rms radii equal to $(2.27 \mathrm{fm})$, while the valence (two-neutron) is assumed to be in a pure $\left(1 \mathrm{p}_{1 / 2}\right)$ with occupation number 0.5 and oscillator size parameter equal to $1.75 \mathrm{fm}$ which gives rms radii equal to $2.55 \mathrm{fm}$. The experimental and calculated nucleon rms radii for this nucleus are displayed in Table 1. It is noted that there is a good agreement between the theoretical calculations and the experimental results. Two body nucleon density distribution (2BNDD'S) in $\mathrm{fm}^{-3}$ of ground state are plotted versus $\mathrm{r}$ (in $\mathrm{fm}$ ) as shown in Fig. 5(a). The black line represents the normal contribution of core ${ }^{6} \mathrm{He}$, the blue line represents the valence (two-neutron exotic nuclei in state of $1 \mathrm{p}_{1 / 2}$ ) through this distribution, it takes the form of a long tail, the red line represents the matter density distribution (core +valence) and it takes the form of long tail too and has a good agreement with the experimental data of ${ }^{8} \mathrm{He}$, which were taken from Antonov [23] and is represented by the shaded area. Fig. 5(b) shows the comparison between the matter density distribution of ${ }^{8} \mathrm{He}$, represented by the red line, and the matter density distribution of stable nuclei ${ }^{4} \mathrm{He}$, represented by the blue line. It is clear from this figure that the red and blue curves are diverse. As the valence two neutrons in ${ }^{8} \mathrm{He}$ are weakly bound, the red curve has a longer tail than that of the blue curve. Fig. 5(a and b) provide the conclusion that the halo phenomenon in ${ }^{8} \mathrm{He}$ is connected to the valence neutrons but not to the core nucleons. 


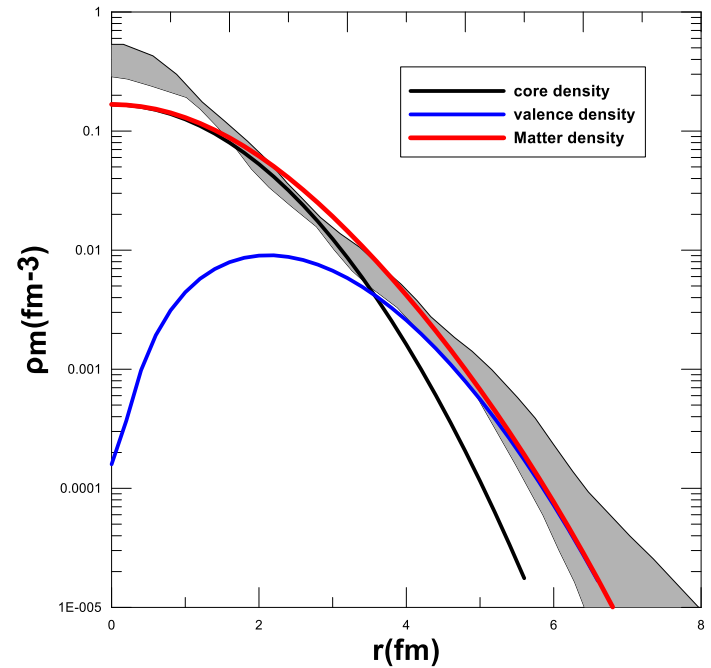

(a)

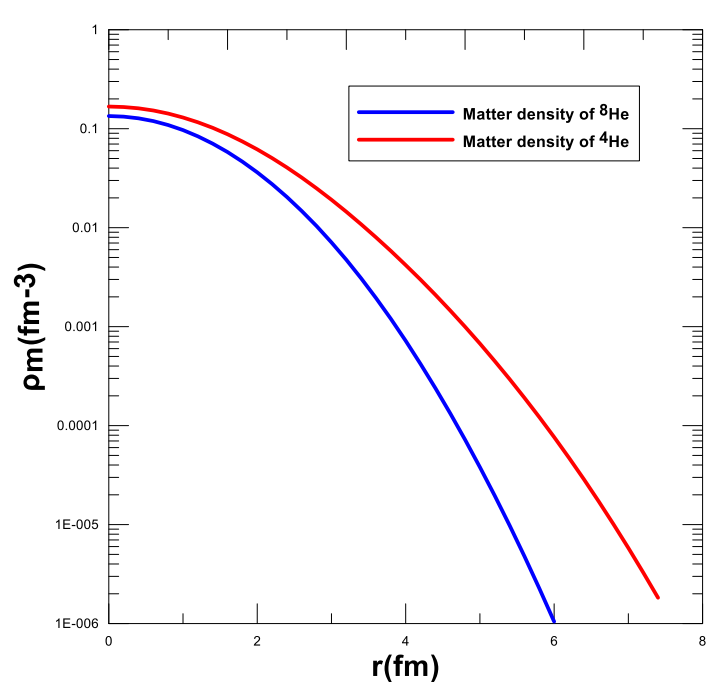

(b)

Figure 5: (a) The comparison between Matter density distributions of ${ }^{8} \mathrm{He}$ with that of experimental data, (b) The comparison between Matter density distributions of ${ }^{8} \mathrm{He}$ and stable ${ }^{4} \mathrm{He}$ nuclei.

The elastic electron scattering form factor 2BCDD's are shown in Fig.6, the red curve represents form factor with oscillator size parameter $(\beta=1.77 \mathrm{fm})$ (assumed to be the average of $\beta_{\mathrm{c}}$ and $\beta_{\mathrm{v}}$ ), while the black filled circles curve represents the experimental data for stable nuclei ${ }^{4} \mathrm{He}$ which were taken from J. S. McCarthy et al. [24]. When comparing the theoretical results with experimental data for stable nuclei $\left({ }^{4} \mathrm{He}\right)$ it was noted that they have the same behavior of first diffraction minimum, Where the first diffraction minimum for ${ }^{8} \mathrm{He}$ is located at $q \approx 2.6 \mathrm{fm}^{-1}$ and for ${ }^{4} \mathrm{He}$ at $q \approx 3.1 \mathrm{fm}^{-1}$.

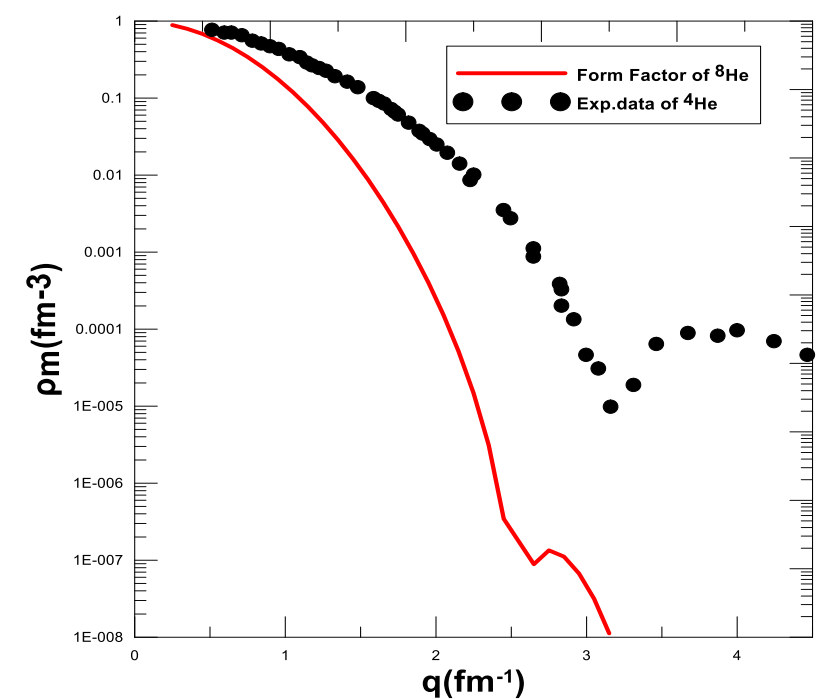

Figure 6: Comparison between the calculated elastic form factors of ${ }^{8} \mathrm{He}$ and the experimental data of stable ${ }^{4} \mathrm{He}$ nuclei.

\section{Conclusions}

Because of two-neutron valence, which are considered to be a distinctive characteristic of halo nuclei, the measured matter density via the framework of two body nucleon density distribution (2BNDD's) with effect of tensor force (TC's) and two different oscillator size parameters $\beta \mathrm{c}$ and $\beta \mathrm{v}$ for our exotic nuclei display a long tail at $(\mathrm{r}>6 \mathrm{fm})$ behavior in this work. The measured matter density and rms radii of $\left({ }^{17} \mathrm{~B},{ }^{11} \mathrm{Li}\right.$, 
${ }^{8} \mathrm{He}$ ) exotic nuclei agrees well with the experimental results. When comparing the theoretical results of elastic form factors for exotic nuclei with experimental data for stable nuclei, it was noted that the location of first diffraction minimum for exotic nuclei was towards the low momentum transfer comparison with the stable nuclei.

\section{Acknowledgements}

The authors thank the head of the Department of Physics and the University of Baghdad, College of Science, Department of Physics for their help during the research work.

\section{Conflict of interest}

Authors declare that they have no conflict of interest.

\section{References}

1. Otsuka T., Exotic nuclei and nuclear forces. Physica Scripta, 2013. 2013(T152): pp. 014007.

2. Tanihata I., Hamagaki H., Hashimoto O., Shida Y., Yoshikawa N., Sugimoto K., Yamakawa O., Kobayashi T., and Takahashi N., Measurements of interaction cross sections and nuclear radii in the light p-shell region. Physical Review Letters, 1985. 55(24): pp. 2676-2679.

3. Abdullah A.N., Matter density distributions and elastic form factors of some twoneutron halo nuclei. Pramana, 2017. 89(3): pp. 1-6.

4. Frederico T., Yamashita M.T., and Tomio L., Structure of large two-neutron halos in light exotic nuclei. Nuclear Physics A, 2007. 787(1-4): pp. 561-568.

5. Frederico T., Universal aspects of neutron halos in light exotic nuclei. Few-Body Systems, 2014. 55(8-10): pp. 651-657.

6. Mahmood R.I., Charge density distributions and electron scattering form factors of 19F, 27Al and 25Mg nuclei. Iraqi Journal of Physics (IJP), 2017. 15(33): pp. 101110.

7. Flaiyh G.N., Inelastic electron scattering form factors involving the second excited $2+$ level in the isotopes 50, 54, 52Cr. Iraqi Journal of Physics (IJP), 2015. 13(28): pp. 19-26.

8. Abdullah A.N., Nuclear matter distributions of neutron rich 6He, 11Li, 14Be and 17B halo nuclei studied by the Bear-Hodgson potential. Modern Physics Letters A, 2020. 35(26): pp. 2050212.

9. Antonov A., Bonev I., Christov C.V., and Petkov I.Z., Generator coordinate calculations of nucleon momentum and density distributions in $4 \mathrm{He}, 16 \mathrm{O}$ and 40 Ca. Il Nuovo Cimento A (1965-1970), 1988. 100(5): pp. 779-788.

10. Lawson R., Theory of the nuclear shell model. Vol. 9. 1980: Clarendon Press Oxford.

11. Fiase J., Hamoudi A., Irvine J., and Yazici F., Effective interactions for sd-shellmodel calculations. Journal of Physics G: Nuclear Physics, 1988. 14(1): pp. 27-36.

12. Radhi R., Hamoudi A., and Ridha A., Elastic Electron Scattering From Unstable Neutron-Rich P. Iraqi Journal of Science, 2013. 54(2): pp. 324-332.

13. Walecka J.D., Electron scattering for nuclear and nucleon structure. Vol. 16. 2001: Cambridge University Press.

14. Brown B., Radhi R., and Wildenthal B., Simultaneous analysis of magnetic moments and elastic magnetic electron scattering form factors. Physics Letters B, 1983. 133(1-2): pp. 5-8.

15. Tanihata I., Kobayashi T., Yamakawa O., Shimoura S., Ekuni K., Sugimoto K., Takahashi N., Shimoda T., and Sato H., Measurement of interaction cross sections 
using isotope beams of $\mathrm{Be}$ and $\mathrm{B}$ and isospin dependence of the nuclear radii. Physics Letters B, 1988. 206(4): pp. 592-596.

16. Fortune H. and Sherr R., Matter radii and wave function admixtures in $2 n$ halo nuclei. The European Physical Journal A, 2012. 48(7): pp. 1-5.

17. Ozawa A., Suzuki T., and Tanihata I., Nuclear size and related topics. Nuclear Physics A, 2001. 693(1-2): pp. 32-62.

18. Alkhazov G., Dobrovolsky A., Egelhof P., Geissel H., Irnich H., Khanzadeev A., Korolev G., Lobodenko A., Münzenberg G., and Mutterer M., Nuclear matter distributions in the $6 \mathrm{He}$ and $8 \mathrm{He}$ nuclei from differential cross sections for smallangle proton elastic scattering at intermediate energy. Nuclear Physics A, 2002. 712(3-4): pp. 269-299.

19. Vaagen J., Gridnev D., Heiberg-Andersen H., Danilin B., Ershov S., Zagrebaev V., Thompson I., Zhukov M., and Bang J., Borromean halo nuclei. Physica Scripta, 2000. 2000(T88): pp. 209.

20. Cichocki A., Dubach J., Hicks R., Peterson G.A., De Jager C., De Vries H., Kalantar-Nayestanaki N., and Sato T., Electron scattering from B 10. Physical Review C, 1995. 51(5): pp. 2406-2426.

21. Alkhazov G., Vorobyov A., Dobrovolsky A., Inglessi A., Korolev G., and Khanzadeev A., Investigation of the structure of light exotic nuclei by proton elastic scattering in inverse kinematics. Physics of Atomic Nuclei, 2015. 78(3): pp. 381386.

22. Suelzle L., Yearian M., and Crannell H., Elastic Electron Scattering from Li 6 and Li 7. Physical Review, 1967. 162(4): pp. 992.

23. Antonov A., Kadrev D., Gaidarov M., de Guerra E.M., Sarriguren P., Udias J., Lukyanov V., Zemlyanaya E., and Krumova G., Charge and matter distributions and form factors of light, medium, and heavy neutron-rich nuclei. Physical Review C, 2005. 72(4): pp. 044307.

24. McCarthy J., Sick I., and Whitney R., Electromagnetic structure of the helium isotopes. Physical Review C, 1977. 15(4): pp. 1396-1414.

\section{كثافة توزيع المادة ومربع نصف القطر وعوامل التشكل للاستطارة الاكترونية لبعض النوى$$
\text { الغريبة( }
$$$$
\text { شيماء علي راهي، غيث نعمه فليح }
$$$$
\text { قسم الفيزياء، كلية العلوم، جامعة بغداد، بغداد، العراق }
$$

تمت در اسه توزيعات الكثافة لبعض نوى الهالة ذات النيوترونين (2BND

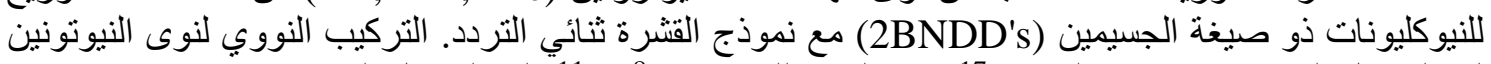

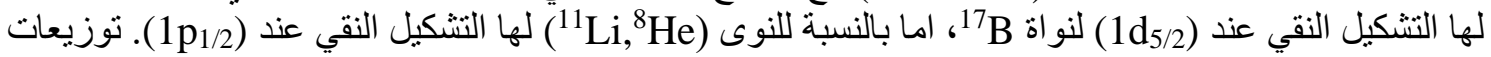

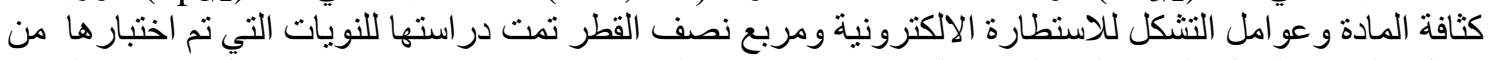

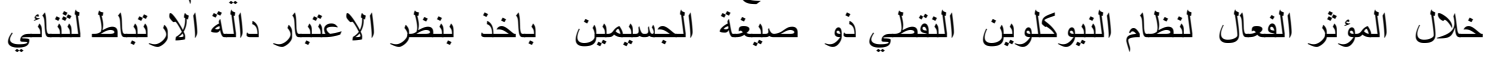

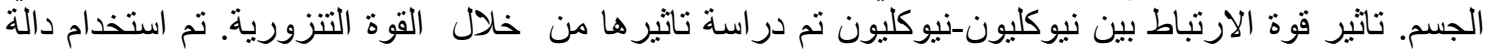

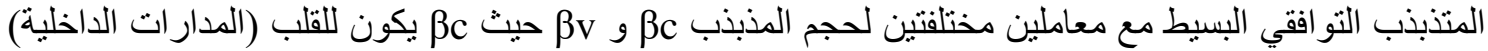

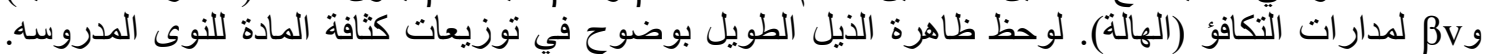

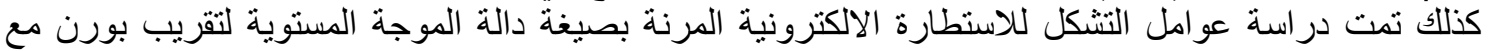

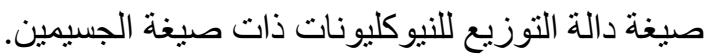

